\title{
A Review of the International FLOSS (Free/Libre Open Source Software) Innovation Surveys
}

Análisis de las encuestas internacionales acerca de innovacione en FLOSS (software libre y de código abierto)

Análise das enquetes internacionais respeito de inovações em FLOSS (software livre e de código aberto)

Hernán Alejandro Morero Facultad de Ciencias Económicas, Universidad Nacional de Córdoba, Argentina. Centro de Investigaciones y Estudios sobre Cultura y Sociedad (CIECS), CONICET y UNC, Argentina.

E-mail: hernanmorero@eco.uncor.edu

Ana Valentina Fernandez Universidad Nacional de Rafaela, Argentina.

E-mail: anavalentinafernandez@unraf.edu.ar
Josefina Sonnenberg Palmieri Universidad Nacional de Rafaela, Argentina. E-mail: josefinasonnenberg@unraf.edu.ar
Fecha de recepción: 10/10/2017 Fecha de aceptación: 12/05/2018

\section{Abstract}

Given the substantial growth that software and IT sector has had in the last decade, it becomes relevant to measure the impact that this expansion has had on the development of emerging economies. Specifically, the study of the FLOSS production activity is relevant given its contribution to the Knowledge Intensive Services Sector. The aim of this study is to design an innovation survey for the software sector that considers the FLOSS activity separately. Moreover, the paper describes an extensive system-

Keywords - Innovation Surveys

- Software firms

- FLOSS

- Developers atization, evaluation and analysis of diverse technological surveys carried out on the software activity and the FLOSS surveys available specified at a firm level, as a way to collect all the possible background which allows proposing a questionnaire that measures the particularities of FLOSS.

\section{Resumen}

A partir del crecimiento sustancial del sector de software y tecnologías de la información en la última década, resulta interesante medir el impacto 
Palabras clave - encuestas sobre innovación - empresas de software

- FLOSS

- desarrolladores

Palabras-chave

- enquetes sobre inovação

- empresas de software

- FLOSS

- desenvolvedores de esta expansión sobre el desarrollo de las economías emergentes. El estudio de la actividad productiva de FLOSS es especialmente relevante debido a su contribución al sector de los servicios intensivos en conocimiento. El objetivo de este trabajo es diseñar una encuesta de innovación para el sector de software que considere a la actividad de FLOSS de manera específica. Además, esta investigación describe la sistematización, la evaluación y el análisis de distintas encuestas tecnológicas realizadas sobre la actividad del software y de las encuestas acerca de FLOSS que están disponibles a nivel empresarial como un medio para proponer, sobre la base de todos los antecedentes que se puedan obtener, un cuestionario que permita medir las particularidades de FLOSS.

\section{Resumo}

A partir do crescimento substancial do setor de software e tecnologias da informação na última década, é interessante medir o impacto desta expansão sobre 0 desenvolvimento das economias emergentes. 0 estudo da atividade produtiva de FLOSS é especialmente relevante devido à sua contribuição ao setor dos serviços intensivos em conhecimento. 0 objetivo deste trabalho é desenhar uma enquete de inovação para o setor de software que considere à atividade de FLOSS de maneira específica. Aliás, esta pesquisa descreve a sistematização, a avaliação e a análise de diferentes enquetes tecnológicas realizadas sobre a atividade do software e das enquetes respeito de FLOSS que estão disponíveis a nível empresarial como um meio para propor, sobre a base de todos os antecedentes que possam se obter, um questionário que permita medir as particularidades de FLOSS. 